\title{
Focusing on mitochondrial form and function
}

\author{
Mitochondria are critical to cellular metabolism, homeostasis and stress responses, and their dysfunction is linked \\ to human disease and pathology. In this issue, we present a Focus of specially commissioned Review articles that \\ discuss recent discoveries and emerging questions in this rapidly advancing field.
}

$\mathrm{M}$ itochondria have long been considered the power stations of the eukaryotic cell, producing the basic unit of cellular energy, ATP, through oxidative phosphorylation. Beyond ATP production, mitochondria maintain ion homeostasis, produce precursors for macromolecules, such as lipids, proteins, and DNA, and generate and sequester potentially damaging metabolic byproducts such as ammonia and reactive oxygen species. In addition, mitochondria are now understood to play active roles in integrating signalling pathways and responses to stressors. Mitochondria are dynamic and their functions have been tightly linked to their fission and fusion, motility, positioning, and form. Recent research has begun to uncover the broad reach of mitochondria in cellular communication through signalling pathways and contacts with other organelles, such as autophagosomes, endoplasmic reticulum, lysosomes and the nucleus. Mitochondrial function and dysfunction have emerged as key factors in mediating metabolic disease, cancer cell metabolism, neurodegeneration and aging, illustrating how mitochondria contribute to health and pathology.

To highlight recent advances and emerging questions in this fast-growing field, we present our Focus on Mitochondrial Form and Function, in which we publish two specially commissioned Review articles by Spinelli and Haigis, on 'The multifaceted contributions of mitochondria to cellular metabolism' (Nat. Cell Biol. https://doi.org/10.1038/s41556-018-0124-1; 2018), and Hajnóczky and co-authors, on 'Mitochondrial dynamics in adaptive and maladaptive cellular stress responses' (Nat. Cell Biol. https://doi.org/10.1038/s41556018-0133-0; 2018). The Focus is hosted on a dedicated page of our website (https://www. nature.com/collections/pptwnffqwc), where readers can also access an online library of related research articles and commentaries published in Nature Cell Biology.

In their Review, Jessica Spinelli and Marcia Haigis discuss the diverse roles of mitochondria in cellular metabolism, including in bioenergetics, macromolecule biosynthesis, nutrient catabolism, redox homeostasis and waste management. They review how mitochondria provide discrete compartments with functions in oxidative metabolism, biosynthetic pathways and signalling, and highlight the importance of compartmentalization to metabolic functions. Pyruvate compartmentalization, glutamine transport and fatty acid oxidation are critical to energy generation. The authors cover how mitochondria participate in the biosynthesis of nucleotides, fatty acids, cholesterol and amino acids, as well as the role of anabolic pathways in cellular stress responses and disease, including cancer. They discuss redox equivalent compartmentalization in the management of $\mathrm{NAD}^{+} / \mathrm{NADH}$ metabolism, and how mitochondria manage metabolic by-products, such as ammonia, reactive oxygen species and hydrogen sulfide, to prevent cellular toxicity and oxidative damage. Unanswered questions remain about metabolite compartmentalization and the authors note that identifying amino acid import/export transporters, will be key as they could act as potential therapeutic targets. Future work will address the physiological contributions of mitochondria through in vivo studies and experiments using physiological metabolite concentrations, and approaches to better understand their contributions to tissue function.

Recent research has uncovered a central role for mitochondrial dynamics in cellular stress responses, a topic reviewed by Hajnóczky and colleagues. These experts discuss how mitochondria sense and respond to physical, acute or chronic stressors, such as genetic alterations in mitochondrial and nuclear genes, mitochondrial fuel substrate deprivation or nutrient oversupply, disturbances in iron and calcium homeostasis, increased reactive oxygen species, or toxins. They cover the central role of mitochondria in supporting survival and stress adaptation, and how mitochondria are integral to programmed cell death signalling to remove damaged cells.

Hajnóczky and colleagues provide a detailed view of mitochondrial motility, reshaping and inter-organellar interactions in response to stressors, and links to pathologies. They discuss the integral role of mitochondrial fission-fusion and recycling to support mitochondrial stability and abundance, distribution, and quality control to allow responses to challenges. The balance of mitochondrial fusion and fission mediates mitochondrial component sharing to support respiration and signalling events, such as calcium oscillations. Mitochondrial fusionfission dynamics are critical to organelle quality control to segregate damaged mitochondria for degradation through mitophagy. Understanding mitochondrial responses to stressors is critical to the transition between health and disease, and studies in genetic models have demonstrated the importance of mitochondrial dynamics in development, neuronal, heart and skeletal muscle function, and neurodegenerative diseases. Future studies in physiological settings will further elucidate the role of mitochondrial dynamics and recycling in specialized tissues, homeostasis and disease.

Studies in model systems have also revealed links between mitochondrial quality control in aging and longevity, as well as roles in calcium homeostasis in skeletal muscle and muscle atrophy. Mitochondria are critical to thermogenesis and adipocyte cellular metabolism, and have emerging roles in glucose homeostasis and metabolic disease. Pathways critical for the maintenance and sensing of mitochondrial DNA have been implicated in inflammation and immune signalling.

Although classic studies have demonstrated the critical role of mitochondria in providing energy for the cell, the diverse functions of this organelle in cellular metabolism and stress responses continue to emerge. Much remains to be elucidated in understanding the functional role of mitochondria in cellular homeostasis and disease states. Nature Cell Biology continues to champion the growing field of mitochondrial biology, and we look forward to publishing future key discoveries in the function and dysfunction of this organelle in cell survival, death, energetics, quality control and homeostasis. With this Focus of commissioned content, and the accompanying library of research articles and commentary from Nature Cell Biology, we aim to provide a perspective on the current trends and unanswered questions driving mitochondrial research. We thank our authors and referees for their contributions, and hope that you will find this Focus on Mitochondrial Form and Function instructive and thought provoking.

Published online: 27 June 2018 https://doi.org/10.1038/s41556-018-0139-7 